\title{
STRESS TENSORS, RIEMANNIAN METRICS AND THE ALTERNATIVE DESCRIPTIONS IN ELASTICITY
}

\author{
J. C. SIMO ${ }^{1}$ and J. E. MARSDEN ${ }^{2}$ \\ University of California, Berkeley.
}

\section{Contents.}

1. Introduction.

2. Some Basic Notation.

3. Alternative Descriptions in Elasticity.

3.1. Material Convected Picture

3.2. Spatial Picture

3.3. Material Rotated Picture

3.4. Lagrangian Picture.

4. A Covariance Approach based on the Balance of Energy Principle •

5. An Example: Isotropic Nonlinear Elastostatics

\section{Introduction}

There are at least four possible alternative pictures useful in the description of the motion of an elastic continuum: the spatial, Lagrangian, convected an rotated pictures. The description of the motion in the rotated picture is obtained essentially by pull-back of the spatial picture with the rotation part of the deformation gradient, as described in Section 2.3.

Our purpose is first to discuss the remarkable duality existing between these alternative descriptions. A key role in describing this duality is played by the spatial formula connecting the spatial metric $g$ and the Cauchy stress

1Post-Doctoral fellow; SESM, UCB.
${ }^{2}$ Dept. of Mathemstics, UCB. 
tensor: $\sigma=\rho \partial \psi / \partial g_{1}$ due to Doyle \& Ericksen [1956]; and its material counterpart connecting the material metric tensor $\mathbf{G}$ and the rotated stress tensor: $\Sigma=\rho \partial \bar{\Psi} / \partial G$, due to Simo \& Marsden [1984]. These formulae illustrate the fact that regardless of the description employed, the stress tensor in that description is obtained by varying the corresponding metric tensor.

Reasons for the importance of these formulae are discussed in Marsden \& Hughes [1983], and Simo \& Marsden [1984]. One of these reasons, the covariance approach based on a covariant formulation of the balance of energy principle, is briefly considered in Section 4.. The essential idea is to extend notions of invariance under superposed spatial isometries that go back at least to Noll [1963]. Toupin [1964], and Green \& Rivlin [1964], to the general notion of invariance under arbitrary spatial diffeomorphisms, which makes elasticity a fully covariant theory.

In most of the continuum mechanics literature, constitutive theory is of ten discussed in terms of the second Piola-Kirchhoff stress tensor. However, some continuum theories capable of including elasticity as a particular case are best formulated in a different picture. Simple examples of this are the notion of hypo-elasticity (Truesdell [1955]. Truesdell \& Noll [1965]), which is formulated directly in the spatial picture, and the generalized hypo-elasticity of Creen \& McInnis [1967] which is formulated in the rotated picture. Another example of practical importance is furnished by most of the computational models employed in finite deformation plasticity, which are often formulated directly in the spatial picture (see e.g., Key \& Krieg [1982]). In this situations, a direct. use of the spatial and material versions of the Doyle-Ericksen formulae in conjunction with the hie derivative results conceptually simpler and often is computationally far more convenient (see Simo \& Pister [1984]). A simple example which illustrates the practical value of these formulae is considered in Section 5.

\section{Some Basic Notation.}

Our notation is summarized as follows. Consider smooth orientable Riemannian manifolds $\left(B, G_{0}\right)$ and $(S, g)$ endowed with Riemannian metrics $\mathbf{G}_{0}$ and $g$, respectively. We speak of $B$ as the fixed reference configuration of the physical body of interest, and we refer to $S$ as the ambient space in which the evolution of the body takes place. Denoting by $C \equiv\left\{\varphi: B \rightarrow S \mid\right.$ a $C^{\infty}$ embedding $\}$ the configuration space, a motion of the 
body is curve of configurations: $t \in \mathbb{R} \rightarrow \varphi_{t} \in C$, and we write $x=\varphi_{t}(X) \equiv \varphi(X, t)$. $X \in B$.

Associated with the motion $\varphi_{\ell}$ one has the material velocity $V_{\ell}: B \rightarrow T S$ defined as $v_{t}(X)=\partial \varphi_{t}(X) / \partial t, X \in B$; and the material acceleration: $A_{l}: B \rightarrow T S$, $A_{t}(X)=\partial V_{t}(X) / \partial t ;$ where we have denoted by $T S$ the tangent bundle on $S$. The spatial velocity $\nabla_{t}: \varphi_{l}(B) \rightarrow T S$ and spatial acceleration $a_{t}: \varphi_{l}(B)->T S$ associated with the motion are defined as $\nabla_{t}=V_{t} \circ \varphi_{t}^{-1}$ and $a_{t}=A_{l} \circ \varphi_{t}^{-1}$.

We denote by $\mathrm{F} \equiv T \varphi_{\ell}: T B \rightarrow T S$ the deformation gradient, and let $\mathbf{C}=\mathrm{F}^{T} \mathbf{F}$ be the right Cauchy-Green Tensors. Employing the standard notation of calculus in manifolds (Lang [1972], Abraham, Marsden \& Ratiu [1983]) we have

$$
\mathrm{C}=\varphi_{i}{ }^{\prime}(\mathrm{g}) \quad \text { i.e., } \quad C_{A B}=F_{A}^{a} F_{B}^{b} g_{a b} \circ \varphi_{t}
$$

Let $\mathbf{G}$ be another metric on $B$ which may be arbitrarily chosen. In particular one may (and often does) take $G=G_{0}$. By the polar decomposition theorem we write

$$
\mathbf{F}=\mathbf{R U} \quad \text { i.e. . } \quad F_{A}^{a}=R_{B}^{a} U_{A}^{B}
$$

where $\mathrm{R}:\left(T_{X} B\right) \rightarrow T_{\varphi_{l}(X)} S$ is a two-point tensor called the rotation tensor, and $\mathrm{U}:\left(T_{X} B\right) \rightarrow\left(T_{X} B\right)$ is the material stretch tensor. Since $\mathrm{R}$ is an orthogonal tensor we have the relations

$$
R_{I}^{a} R_{J}^{b} g_{a b} \circ \varphi_{t}=G_{I J}, \quad R_{A}^{I} U_{B}^{J} G_{I J}=C_{A B}
$$

To emphasize the geometric meaning, relations (2.3) will be written employing a pull-back/push-forward notation as

$$
\mathbf{G}=\mathbf{R}^{*}(\mathrm{~g}), \quad \mathbf{C}=\mathbf{U}^{*}(\mathrm{G})
$$

One should carefully note that the right Cauchy Green tensor $\mathbf{C}$ can be regarded either as a function of $F$ and $g$ through representation (2.1), or as a function of $\mathbf{U}$ and $\mathbf{G}$ through representation $(2.4)_{2}$. Indeed, the former point of view leads to spatial Doyle-Ericksen formula. Doyle \& Ericksen [1956], whereas the latter yields the material version of this formula. 


\section{Alternative Descriptions in Elasticity.}

In this section we shall consider the possible alternative descriptions of the motion of a continuum: the spatial, material Lagrangian, material convected and material rotated descriptions. Confining our attention to non linear elasticity, our purpose is to emphasize the duality that exists between these four possible alternative pictures of the given motion. In describing this duality, the formula first derived by Doyle \& Ericksen [1956] connecting (Cauchy) stress tensor and spatial metric is crucial for understanding the spatial description. The material version of this formula plays the dual role in the rotated description and completes the duality between the four alternative pictures of the motion.

\subsection{Convected Material Picture.}

First, recall that associated with a given motion $t \rightarrow \varphi_{t} \in C$ one defines the convected objects by pulling-back their spatial counterparts to the reference configuration $\left(B, G_{0}\right)$. Accordingly, the convected velocity $v_{t}$ and convected acceleration $\alpha_{\ell}$ are vector fields on $B$ defined as

$$
\nu_{l}=\varphi_{i}^{i}\left(\nabla_{l}\right) . \quad \alpha_{l}=\varphi_{i}^{i}\left(a_{l}\right)
$$

We note that if $T \equiv J \sigma$ denotes the Kirchhoff stress tensor and $S$ is the symmetric Piola-Kirchhoff stress tensor, then $\mathbf{S}=\varphi_{i}(\tau)$, and hence $\mathbf{S}$ and $\mathbf{C}=\varphi_{i}(\mathrm{~g})$ are simply the convected (Kirchhoff) stress tensor and convected metric tensor.

Next, recall that for a thermoelastic material the free energy function $\psi$ depends on the motion locally through the point values of $\mathrm{C}=\varphi_{i}{ }^{\circ}(\mathrm{g})$ (Coleman \& Noll [1959]). Following standard abuse of notation we write $\Phi\left(X, C(X), \Theta(X), \mathrm{G}_{0}(X)\right)$ for this dependence. The classical constitutive equation for the stress tensor $\mathbf{S}$ then takes the form

$$
S=2 \rho_{R \in f} \frac{\partial \Phi}{\partial C}
$$

where $\rho_{R f}$ is the mass density in the reference configuration. Equation (3.2) is nothing but the relation connecting stress tensor, energy density and spatial metric expressed in the convected picture, as the discussion of the spatial and rotated pictures will clearly reveal. The rate form of (3.2) is

$$
\dot{\mathbf{S}}=\mathrm{C}: \dot{\mathbf{C}}+m: \dot{\Theta}
$$


where $C \equiv 2 \rho_{\text {Rof }} \frac{\partial^{2} \Phi}{\partial C \partial C}$ is the material second elasticity tensor, and $m \equiv 2 \rho_{\text {Raf }} \frac{\partial^{2} \Phi}{\partial C \partial \theta}$ the material thermal coefficients.

Remark: One must include $G_{0}$ as an argument in $\Phi$ since it is needed to form scalars from C; e.g.. $\operatorname{tr} \mathrm{C}=C_{A B} G_{0}^{A B}$

\subsection{Spatial Picture.}

Associated with the motion $t \rightarrow \varphi_{t} \in C$ one has in the spatial description the spatial velocity $\nabla_{\ell}$, the spatial acceleration $a_{\ell}$ and the Cauchy stress tensor $\sigma$. It might appear somewhat surprising that to complete the spatial description one must also include the spatial metric tensor $g$. This need for including the metric tensor was first recognized by Doyle and Ericksen [1956], and may be motivated as follows.

Since $\mathbf{C}=\varphi_{8}^{\circ}(\mathbf{g})=\mathrm{F} \cdot \mathrm{g} \cdot \mathrm{F}$, the right Cauchy-Crcen tensor $\mathbf{C}$ depends parametrically on the metric g. As a result, the spatial free energy $\psi$ defined by $\mathscr{W}\left(x, g, F, \Theta, G_{0}\right)=\Phi\left(X, \varphi_{i}{ }^{\prime}(g), F, \Theta, G_{0}\right)$ depends on $g$ and this dependence, as first recognized in Doyle \& Ericksen [1956], must be tensorial. Indeed, a simple argument involving the chain rule (see e.g.. Marsden \& Hughes [1983]) shows the equivalence between the classical formula (3.2) and the following spatial formula:

$$
\sigma=2 \rho \frac{\partial \Psi}{\partial g}
$$

Formula (3.4) puts in evidence the fact that the spatial stress tensor is in fact obtained by varying the internal energy with respect to the spatial metric tensor g. Notice that formula (3.2) responds to the same concept although expressed in a different picture.

In applications concerned with inelastic behavior it is often necessary to consider the rate form of (3.2); a typical example being rate independent finite deformation plasticity.

Rate Constitutive Equation. The rate form of the spatial formula (3.4) involves measuring the rate of change of the stress tensor $\sigma$ relative to the $f$ low of spatial velocity field $\nabla_{t}$; this flow is given by $\gamma_{l, s}=\varphi_{s} \circ \varphi_{l}^{-1}: \varphi_{l}(B) \rightarrow \varphi_{s}(B)$. The standard way of forming rates is to employ the notion of Lie derivative (see e.g.. Abraham. Marsden \& Ratiu [1983]). For any spatial tensor field $t_{t}$. its Lie 
derivative relative to the flow $\gamma_{t, s}=\varphi_{s} \circ \varphi_{l}^{-1}$ is defined by

$$
L_{\nabla}\left(t_{t}\right)=\left.\frac{d}{d s}\right|_{s=t}\left(\gamma_{t, s} t_{s}\right) \equiv \varphi_{t} *\left(\frac{\partial}{\partial t} \varphi_{t}^{\prime}\left(t_{t}\right)\right)
$$

In particular, for the metric tensor g one has the following key formula:

$$
\operatorname{Lr}(g)=\varphi_{t} *(\dot{C}) \equiv 2 d
$$

where $\mathrm{d} \equiv / / 2 \varphi_{t}{ }_{*}(\dot{\mathrm{C}})$ is the spatial rate of deformation lensor. By applying the Lie derivative to both sides of the Doyle-Ericksen formula (3.4) we are led to the following rate constitutive equation:

$$
\frac{1}{J} I_{v}(J \sigma) \equiv c: \mathbf{d}+\mathbf{m} \cdot \dot{\oplus}
$$

One calls $c=4 \rho \frac{\partial^{2} \psi}{\partial g \partial g}$ the spatial second elasticity tensor, and one refers to $\mathbf{m}=2 \cdot \rho \frac{\partial^{2} \psi}{\partial \mathrm{g} \partial \theta}$ as the spatial thermal stress coefficients. $\sigma \equiv \mathrm{I}_{\boldsymbol{\gamma}}(\tau) / J \equiv$ is known as the Truesdell rate of Cauchy stresses.

\subsection{Material Rotated Descriplion.}

If one introduces the polar decomposition: $F=R U$, an alternative description of the motion $t \rightarrow \varphi_{\ell} \in C$ is obtained by R-rotating the spatial objects (fields on $\varphi_{t}(B)$ ) back to the reference configuration $B$. The rotated velocity $V_{t}^{R}$ and rotated acceleration $A^{R}$ are thus vector fields on $B$ defined as

$$
\mathbf{V}^{R}=\mathrm{R}^{*}\left(\mathbf{v}_{t}\right), \quad \quad \mathbf{A}^{R}=\mathrm{R}^{*}\left(\mathbf{a}_{t}\right)
$$

The rotated stress tensor $\Sigma$ is defined in the obvious manner by setting $\Sigma=R^{*}(\sigma)$. To complete the description in the rotated picture one needs to introduce the metric tensor $G=R^{*}(g)$. The reason for this is that, since $C=U^{*}(U)$, the free energy $\bar{\Psi}$ in the rotated picture may be defined as $\bar{\Psi}\left(X, \mathbf{G}, \mathbf{U}, \Theta, \mathbf{G}_{0}\right) \equiv \Phi\left(X, \mathbf{U}^{*}(\mathbf{G}), \Theta, \mathbf{G}_{0}\right)$. Indeed, a argument involving the chain rule shows that the material formula (3.2) or the spatial Doyle-Ericksen formula (3.4) are equivalent to the following formula

$$
\Sigma=2 \rho \frac{\partial \bar{\Psi}}{\partial \mathbf{G}}
$$


To emphasize the duality between the spatial and the material rotated pictures we consider the rate form of constitutive equation (3.9) which may be regarded as the material version of the Doyle-Ericksen formula (3.4).

Rate Constitutive Equation. Let us introduce the material stretch Lie derivative by formally replacing pull-back/push forward operations with the deformation gradient $F$ in definition (3.5) with the stretch part $U$ of $F$. That is, for any material tensor fleld $T_{1}$, define its material stretch lie derivative as

$$
\operatorname{Lu}_{\mathrm{J}}\left(\mathrm{T}_{t}\right)=\mathrm{U}_{*}\left(\frac{\partial}{\partial t} \mathrm{U}^{*}\left(\mathrm{~T}_{t}\right)\right)
$$

It can be shown that definition (3.10) is simply the Lie derivative with respect to the rotated velocity field $\mathbf{v}^{R}=\mathbf{R}^{*}\left(\mathbf{v}_{t}\right)$ or, equivalently, the $R$-rotated lie derivative. The motivation this definition is that by applying (3.10) to the metric tensor $G$ we obtain the following formula dual to (3.6):

$$
L_{0}(G)=U_{*}(\dot{C})=2 U_{*}\left(\varphi_{i} \dot{d}\right)=2 R^{*}(d) \equiv 2 \Lambda
$$

where $\Lambda \equiv R^{*}(d) \equiv$ is the rotated rate of deformation tensor. By applying the material stretch Lie derivative to both sides of formula (3.9) one obtains the following rate constitutive equation dual to (3.7):

$$
\frac{1}{J} \operatorname{LQ}(J \Sigma) \equiv \Xi: \Lambda+M \cdot \dot{\Theta}
$$

We refer to $\Xi \equiv 4 \rho \frac{\partial^{2} \Psi}{\partial G} \frac{\bar{\Psi}}{\partial G}$ as the rotated second elasticity tensor, and to $\mathbf{U} \equiv 2 \frac{\partial^{2} \Psi}{\partial \mathbf{G}} \frac{}{\partial \Theta}$ as the rotated thermal stress coefficients. $\mathbf{T} \equiv J \boldsymbol{\Sigma} \equiv \mathbf{R}^{*}(\tau)$ is simply the rotated Kirchhoff stress tensor.

Finally, we consider the most commonly employed description of the

motion in nonlinear elasticity.

\subsection{Material Lagrangian Picture.}

In the Lagrangian description, the motion is characterized by the material velocity $V_{l}: B \rightarrow T S$ and material acceleration $A_{l}: B \rightarrow T S$. As noted in Section 2 , these are vector fields covering $\varphi_{l}: B \rightarrow S$ obtained from their spatial counterparts $\nabla_{t}$ and $a_{t}$ by composition with the motion $\varphi_{t}$. Similarly, tensor fields 
characterizing the motion become two-point tensor obtained from their spatial counterparts by partial pull-back. Thus, the stress tensor in the Lagrangian description becomes the non-symmetric Piola-Kîrchhoff tensor: $\mathrm{P}^{*} \equiv J(\sigma \circ \varphi) \mathrm{F}^{-T} \uparrow$, and in the Lagrangian description the stress-stored energy relation takes the classical form

$$
\mathbf{p}^{t}=\rho_{R \theta f} \frac{\partial \overline{\bar{\Psi}}}{\partial \mathrm{F}^{\circ}}
$$

We note that this formula is consistent with the spatial Doyle-Ericksen formula (3.4). In fact, formula (3.13) may be obtained directly from (3.4) through a chain rule argument, by noting that $F^{b} \equiv\left(\right.$ bo $\left.\varphi_{l}\right) F$ plays the same role as that played by $\mathrm{g}^{6}$ in the spatial picture.

For convenience and comparison purposes, the variables entering in the four descriptions discussed in this section together with the particular form taken by the stress-stored energy relation, have been collected in TABLE 1. below. It should be noted that the representation for the stress tensor in the alternative descriptions may be all thought of as a particular case of the spatial Doyle-Ericksen formula.

Remark. The covariant argument described in Section 4 shows that the spatial free energy $\Psi$ in the spatial picture depends on $F$ only through its rotation part $\mathbb{R}$. That is one has: $\Psi\left(x, R_{1}, G_{0}\right)$ in the spatial picture, and $\Psi\left(X, U, G, G_{0}\right)$ in the rotated picture.

The symbol (.) indicates "contravariant" components (indices up), whereas (. ) ${ }^{6}$ indicates "covariant" components (indices down). See e.g., Marsden \& Hughes [1883], Sect. 1.4. 
TABLE 1.

Alternative Descriptions: Variables Involved.

\begin{tabular}{|c|c|c|c|}
\hline Spatial & Convected & Rotated & Lagrangian \\
\hline$\nabla_{i}$ & $\nu_{t}=\varphi_{i} \nabla_{t}$ & $\mathbf{v}_{R_{t}}=\mathrm{R}^{*} \mathbf{v}_{t}$ & $v_{t}=v_{t} \circ \varphi$ \\
\hline$a_{t}$ & $\alpha_{i}=\varphi_{i}^{*} a_{i}$ & $A_{R_{l}}=R^{*} a_{l}$ & $A_{t}=a_{t} \circ \varphi$ \\
\hline$g^{6}$ & $C^{b}=\varphi_{i}^{i} g^{b}$ & $G^{b}=R^{*} g^{b}$ & $F^{b}=\left(g^{b} \circ \varphi\right) F$ \\
\hline$\sigma^{*}$ & $S^{t}=J \varphi_{i}^{*} \sigma^{*}$ & $\Sigma^{*}=\mathrm{R}^{*} \sigma^{*}$ & $P^{*}=\left(\sigma^{*} \circ \varphi\right) F^{-T}$ \\
\hline$\sigma=2 \rho \frac{\partial \psi}{\partial g}$ & $\mathrm{~S}=2 \rho_{R \theta \rho} \frac{\partial \Phi}{\partial \mathrm{C}}$ & $\Sigma=2 \rho \frac{\partial \bar{\Psi}}{\partial \mathbf{G}}$ & $\mathbf{P}=\rho_{\text {Rog }} \frac{\partial \overline{\bar{\Psi}}}{\partial \mathbf{F}}$ \\
\hline$c=4 \rho \frac{\partial^{2} \Psi}{\partial g \partial g}$ & $C=4 \rho_{R o f} \frac{\partial^{2} \Phi}{\partial C \partial C}$ & $\ddot{\Xi}=4 \rho \frac{\partial^{2} \bar{\Psi}}{\partial G \partial G}$ & $\mathrm{~A}=\rho_{\text {Rof }} \frac{\partial^{2} \overline{\bar{\Psi}}}{\partial \overline{\mathrm{F}} \partial \overline{\mathrm{F}}}$ \\
\hline
\end{tabular}

\section{Covariant Formulation Based on Balance of Fnergy.}

At least two procedures can be employed to formulate elasticity as a fully covariant theory. One can make use of the Hamiltonian formalism and proceed either materially [Marsden \& Hughes 1983] or spatially [Marsden, Ratiu \& Weinstein 1983]. Alternatively, one may base the formulation on a covariant version of the balance of energy principle. In this section we shall focus on some of the aspects involved in the later procedure. Reasons for the importance of the covariance approached based on a covariant balance of energy principle are discussed in Simo \& Marsden [1984] and Marsden \& Hughes [1983]. Our purpose here is to emphasize the duality between the spatial and rotated pictures which is clearly put in evidence through the covariant argument.

The essential idea behind the covariant approach is to extend the balance of energy principle to hold, not only for superposed spatial isometries as stated in Green \& Rivlin [1964], but for superposed arbitrary diffeomorphisms. To achieve this invariance one introduces, in addition to the balance of energy principle, a covariance assumption on how this principle must hold for a given motion. Summarized below are these two basic ingredients.

(i) Balance of Energy: Consider a fixed motion $\varphi_{l}: B \rightarrow S$, and let $\Omega \subset B$ be any compact region with smooth boundary $\partial \Omega$. In addition, let $b(x, t)$ be the 
external body force per unit of mass, $t(x, t, n)$ the Cauchy traction vector. $e(x, t)$ the internal energy per unit of mass, $r(x, t)$ heat supply per unit mass and $h(x, t, \mathbf{n})$ heat $f l u x ; \mathbf{n}(x)$ being the normal to the boundary $\partial \varphi_{\ell}(\Omega)$. We say that balance of energy holds if:

$$
\frac{d}{d t} \int_{\nabla_{t}(\Omega)} \rho\left(e+\not k\left\langle\nabla_{t}, \nabla_{t}\right\rangle\right) d v=\int_{F_{t}(n)} \rho\left\langle\left\langle b_{1} \nabla_{t}\right\rangle+r\right) d v+\int_{\theta_{t} t_{l}(n)}\left(\left\langle t_{1} \nabla_{t}\right\rangle+h\right) d s(4.1)
$$

(ii) Covariant Assumption: For the fixed motion $\varphi_{l}: B \rightarrow S$ satisfying (4.1), consider an arbitrary spatial diffeomorphisms $\xi_{\ell}: S \rightarrow S$, and postulate that the new motion $\bar{\varphi}_{t}=\xi_{t} \circ \varphi_{t}$ also satisfies the balanse of energy equation (4.1) provided: (a) velocities, forces and accelerations are transformed according to the standard dictates of the (Cartan) theory of the classical spacetime, and (b) the metric $\mathrm{g}$ is replaced by $\xi_{\mathrm{i}} \mathrm{g}$.

Thus, the crucial part of the covariant assumption is that the internal energy must depend tensorially on the metric $g$ and, consequently, transform according to

$$
\bar{e}(\bar{x}, t, g)=e(x, t, \xi i g) . \quad \bar{x}=\xi_{l}(x)
$$

For a justification of this tensorial dependence see Simo \& Marsden [1984], and for background motivation on this covariance assumption consult Marsden \& Hughes [1983].

As in the Hamiltonian approach, with the covariance assumption at hand one may now proceed either spatially or materially. To put in evidence the duality between both approaches we review the basic constructions involved in terms of the polar decomposition.

Spatial Picture: The basic idea is to evaluate the balance of energy equation (4.1) for the given motion $\varphi_{t}: B \rightarrow S$ and for the superposed motion $\bar{\varphi}_{t}=\xi_{t} \circ \varphi_{t}$ with the change in metric resulting from the covariance assumption accounted for.

As in the Green-Rivlin argument, use of the transport theorem, the divergence theorem and the Cauchy tetrahedron construction yields the laws of motion. However, since one now considers not only isometries but arbitrary dif feomorphisms, the equality $\dot{e}=\dot{\bar{s}}$ can no longer hold. Indeed, if it were true the stress tensor would vanish identically. In the present argument, use of the covariance assumption and the definition Lie derivative yields, on account of 
the arbitrariness of $L_{w}(g) \pi$, the additional condition:

$$
\sigma=2 \rho \frac{\partial e}{\partial g}
$$

That is, the spatial Doyle-Ericksen formula emerges as the crucial condition which serves the purpose of relaxing the "rigidity" of the covariant assumption demanding that balance of energy must hold under superposed arbitrary spatial diffeomorphisms.

In terms of the polar decomposition the argument just outlined amounts to the construction summarized in the following diagram:

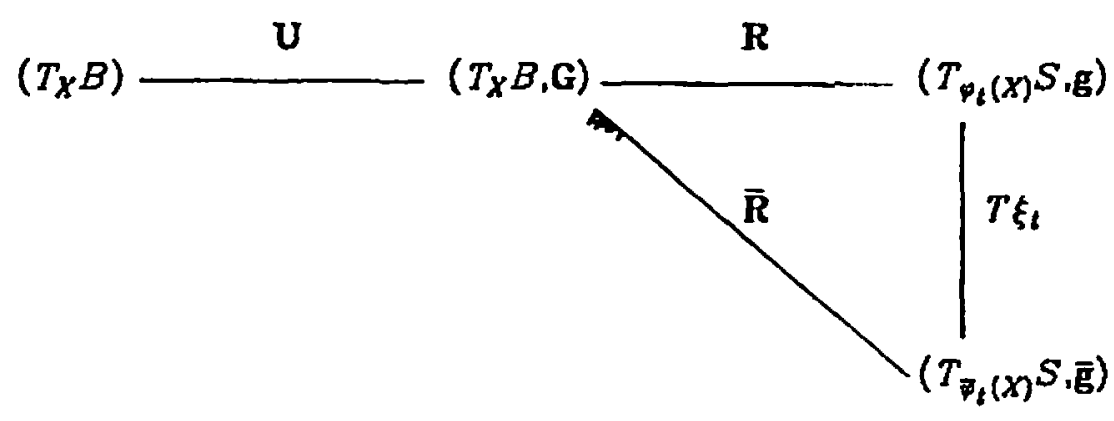

We note that:

$$
\overline{\mathbf{E}}=\xi_{t}(\mathrm{~g}) . \quad \overline{\mathbf{R}}=T \xi_{l} \circ \mathbf{R}_{\circ} \xi_{t}^{-1}, \quad \overline{\mathbf{U}} \equiv \mathbf{U}
$$

Thus, the metric $\mathbf{G}$ and and the stretch tensor $U$ remain unchanged through the argument and, as a result, so does $C=U^{*}(G)$. Only the rotation tensor $R$ is changed by the superposed spatial diffeomorphism.

Material Rotated Picture In the rotated description of the motion we allow G to change with superposed spatial diffeomorphisms by introducing a construction dual to that summarize above. Accordingly, we now hold the rotation tensor $\mathbf{R}$ fixed while $U$ and $G$ change in a way that leaves $C$ unchanged. One is then led to the situation summarized in the following diagram in terms of the polar decomposition.

$$
\begin{array}{lllll} 
& \mathbf{U} & \mathbf{R} & \left(T_{\varphi_{t}(X)} S, \mathbf{g}\right)
\end{array}
$$

Here $w: \varphi_{t}(B) \rightarrow T S$ is defined as $w=\left.\frac{d}{d t}\right|_{\ell=t_{0}} \xi_{t}$. where $t=t_{0}$ is chosen so thet $\left.\xi_{1}\right|_{t=t_{0}}=$ identity. 


$$
\begin{array}{lll}
\left(T_{X} B, \overline{\mathrm{G}}\right) & \mathbf{R} & \left(T_{\nabla_{l}(X)} S, \overline{\mathrm{E}}\right)
\end{array}
$$

where, $H$ is such that

$$
\mathrm{H}=\mathrm{R}^{-1} \circ T \xi_{t} \circ \mathrm{R} \circ \varphi_{i}^{-1} \equiv \mathrm{R}^{*} \circ \xi_{l} *
$$

One should carefully note that although the metric G transforms tensorially, the metric $G_{0}$ in reference configuration remains unchanged.

If we define the material form $E(X, t, \mathrm{G})$ of the internal energy in the obvious tensorial manner by setting

$$
E(X, t, G)=e\left(\varphi_{t}(X), t, R_{*}(\mathrm{G})\right) .
$$

an argument analogous to that of the spatial picture again yields the laws of motion. In addition, use of the covariance assumption in conjunction with the definition of material stretch Lie derivative introduced in Section 2.3 leads, on account of the arbitrariness of $L_{\pi}(G) \pi$, to the condition:

$$
\Sigma=2 \rho \frac{\partial E}{\partial \mathrm{G}},
$$

which is the formula dual to the spatial formula (4.2) in the the rotated description. The duality between both pictures is thus complete.

Remarks: (1) Note that the formula dual to to formula (4.2) in the convected description can be obtained as a particular case of the construction developed in the rotated picture by adopting a particular choice of metric G. By chosing $\mathrm{G}(X)=\varphi_{i}{ }^{\prime}(\mathrm{g}) \equiv \mathrm{C}(X)$; i.e., the convected metric, one obtains the formula:

$$
\mathbf{S}=\frac{2}{J} \frac{\partial E}{\partial C}
$$

(2) Our material covariant argument is a material formulation of the notion of invariance under superposed spatial diffeomorphisms. It does not involve, nor does it imply, the assumption of material covariance which embodies the notion of invariance under superposed material diffeomorphisms. (See

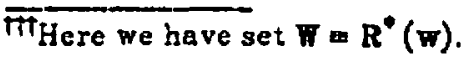


Simo \& Marsden [1984]).

\section{A Simple Example: Isotropic Nonlinear Elastostatics.}

As a simple example which further illustrates the complete duality existing between spatial and rotated pictures through the two versions of the DoyleEricksen formula, we consider the case of isotropic elastostatics. Our purpose is to illustrate the conceptual simplicity and computational convenience of a direct formulation employing the Lie Derivative \& the Doyle-Ericksen formulae. In situations where representation theorems are sought in a specific picture, typically the spatial picture, it is conceptually more clear and computationally far more convenient to proceed directly rather than constantly refer back to a convected representation in terms of the second Piola Kirchhofi stress tensor. Thus, although not often recognized, the spatial and material Doyle-Fricksen formulae make the direct development of constitutive theory in the spatial or rotated pictures as easy as in the often favored convected picture.

Spatial Picture: A simple argument involving material frame indifference shows that the stored energy function for a isotropic material depends on the invariants of the left Cauchy-Green tensor $b^{*}=\varphi_{t}\left(G^{*}\right)$. To develop a representation for $\sigma$ first note that

$$
L_{v}\left(b^{t}\right) \equiv \varphi_{t} * \frac{\partial}{\partial t} \varphi_{i} b^{*} \equiv \varphi_{t} * \frac{\partial}{\partial t} \varphi_{i}^{*} G^{\prime} \equiv 0
$$

A direct application of the definition of Lie derivative then yields for the invariants $I_{b}$. $I_{b}$. III $b$ the formulae

$$
\begin{aligned}
& L_{w}\left(I_{b}\right) \equiv I_{n}\left(b^{t}: g^{b}\right) \equiv 2 b^{t}: d \\
& I_{r}\left(I_{b}\right) \equiv / 2 I_{\nabla}\left(I_{b}^{2}-\left[b^{6}\right]^{2}: g^{b}\right) \\
& =2\left[I_{b} b^{4}-\left(b^{1}\right)^{2}\right]: d \equiv 2\left[1 I_{b} g^{t}-I I J_{b} b^{-1}\right]: d
\end{aligned}
$$

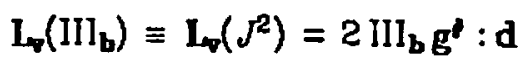

Thus, defining: $W\left(x, I_{b}, I_{b}, I I I_{b}\right) \equiv \rho \psi(x, F, g)$, from the rate form of the spatial Doyle-Ericksen formula

$$
\rho \dot{\Psi}=\sigma: \mathbf{d} \equiv \rho \frac{\partial \Psi}{\partial \mathrm{g}}: \mathrm{L}_{\boldsymbol{\gamma}}(\mathrm{g}) \equiv \rho \mathrm{L}_{\boldsymbol{\nabla}}(\tilde{\psi})
$$


the classical result follows directly:

$$
\sigma \equiv \frac{2}{J}\left\{\left[\mathrm{II}_{\mathbf{b}} \frac{\partial W}{\partial \mathrm{II}_{\mathbf{b}}}+\mathrm{II}_{\mathbf{b}} \frac{\partial W}{\partial \mathrm{II}_{\mathbf{b}}}\right] \mathbf{g}^{\prime}+\frac{\partial W}{\partial \mathrm{I}_{\mathbf{b}}} \mathbf{b}^{*}-1 \mathrm{II}_{\mathbf{b}} \frac{\partial W}{\partial \mathrm{II}_{\mathbf{b}}} \mathbf{b}^{-1}\right\}
$$

Remarks: (1) One can carry out exactly the same argument directly in the rotated picture. The essential observation, dual to (5.1), is that

$$
\mathrm{L}_{\mathrm{U}}\left(\mathrm{C}^{*}\right) \equiv \mathrm{U}_{*} \frac{\partial}{\partial t} \mathrm{U}^{*} C^{*} \equiv \mathrm{U}_{*} \frac{\partial}{\partial t} \mathrm{U}^{*} \mathrm{G}^{*} \equiv 0
$$

and all that is needed is to replace $I_{v}(\cdot)$ by $L_{\mathcal{U}}(\cdot), \sigma$ by $\Sigma$ and $b^{*}$ by $C^{*}$ in equations (5.2) to (5.4).

(2) By this type of direct calculations one can, for example, show that a nonlinear material with constant, isotropic spatial elasticities c for all possible configurations $\in \in C$ cannot be elastic (Simo \& Pister [1984]). Indeed such a material furnishes a non-trivial example of a hypo-elastic material in the sense of Truesdell, which is not elastic. This result and related results in the rotated picture are specially relevant to finite deformation plasticity theories.

\section{References}

ABRAHAM, R., J.E. MARSDEN \& T. RATIU, 1983. Manifolds, Tensor Analysis and Applications, Reading, MA: Addison-Wesley Publishing Co.

COLEMAN, B.D., and W. NOLL, 1959. "On the Thermodynamics of Continuous Media," Arc. Rat. Mech. An., 4, pp.97-128.

DOYLE , T. C. and J. L. ERICKSEN, 1956. Nonlinear Elasticity, in Advances in Applied Mechanics IV. New York: Academic Press, Inc.

GREEN, A.E. and B.C. McINNIS, 1967. "Generalized Hypo-Elasticity." Proc. Roy. Soc. Edinburgh, A57, 220.

GREEN. A.E. and R.S. RIVLIN, 1964a. "On Cauchy's Equations of Motion," J. Appl. Math. and Physics (ZAMP), 15. pp.290-292.

KEY, S.W. \& R.D. KRIEG, 1982. "On the Numerical Implementation of Inelastic Time Dependent and Time Independent, Finite Strain Constitutive Equations in Structural Mechanics," Comp. Meth. Appl. Mech. Engn., 33, pp.439452. 
LANG, S., 1972. Differential Manifolds . Reading, MA: Addison-Wesley Publishing Co. Inc.

MARSDEN, J.E. and T.J.R. HUGHES, 1983. Mathematical Foundations of Elasticity, Englewood-Cliffs, NY: Prentice-Hall, lnc.

MARSDEN, J.E., T. RATIU, and A. WEINSTEIN, 1982. "Semidirect Products and Reduction in Mechanics," Trans. Am. Math. Soc., (to appear).

NOLL, W., 1963. La Methode Axiomatique dans les Mecaniques Classiques et Nouvelles, Paris, pp.47-56.

SIMO, J.C. \& J.E. MARSDEN, 1984. "On the Rotated Stress Tensor and the Material Version of the Doyle-Ericksen Formula," Arc. Rat. Mech. An., (To appear)

SIMO, J.C., \& K.S. PISTER, 1984. "Remarks on Rate Constitutive Equations for Finite Deformation Problems," (Preprint)

TOUPIN, R.A., 1964. "Theories of Elasticity with Couple Stress," Arc. Rat. Mech. An., 11, pp.385-414.

TRUESDELl, C. 1955. "Hypo-elasticity." J. Rational Mechanics Mech. Anal., 4, pp.83-133.

TRUESDELL, C. and W. NOLL, 1965. The Non-Linear Field Theories of Mechanics. in Handbuch der Physit, Vol.Ill/3. S. Flugge, ed., Berlin: Springer-Verlag. 